\title{
EXPLORING THE DRIVING BEHAVIOR OF YOUTH WITH AN AUTISM SPECTRUM DISORDER: A DRIVER INSTRUCTOR QUESTIONNAIRE
}

\author{
Veerle Ross ${ }^{\mathrm{a}}$, Ellen M. M. Jongen ${ }^{\mathrm{a}}$, Marleen Vanvuchelen ${ }^{\mathrm{b}}$, Tom Brijs ${ }^{\mathrm{a}}$, Kris Brijs ${ }^{\mathrm{ac}}$, Geert Wets ${ }^{\mathrm{a}}$ \\ a Transportation Research Institute (IMOB), Hasselt University \\ ${ }^{\mathrm{b}}$ Faculty of Medicine, and Life Sciences, Hasselt University \\ ${ }^{\mathrm{c}}$ Faculty of Applied Engineering Sciences, Hasselt University \\ Diepenbeek, Belgium \\ Email: \{veerle.ross; ellen.jongen, marleen.vanvuchelen; tom.brijs, kris.brijs, \\ geert.wets\}@uhasselt.be
}

\begin{abstract}
Summary: Youth with an autism spectrum disorder (ASD) depend to a great extent on friends and family for their transportation needs. Although little research exists, Cox et al. (2012) surveyed parents/caregivers of youth with ASD (previously) attempting to learn to drive. This study serves as an extension by surveying driver instructors. Several questions queried advice for teaching youth with ASD how to drive, and for improving the current driving education to better fit the needs of youth with ASD. Furthermore, respondents were asked to indicate whether specific characteristics, often associated with ASD, have an impact on driving ability. A total of 52 driver instructors reported potential problems when teaching youth with ASD to drive. Advice for teaching youth with ASD to drive mainly focused on a need for structure, clarity, visual demonstration, practice, repetition and an individualized approach. Results however also showed that the relation between ASD and driving performance might not always be negative but can be positive. Practical implications are provided.
\end{abstract}

\section{INTRODUCTION}

Driving allows autonomy and permits maintenance of social- and work-related contacts (Cox, Reeve, Cox, \& Cox, 2012; Reimer et al., 2013). Nevertheless, youth with an autism spectrum disorder (ASD) depend to a great extent on friends and family for their transportation needs (Feeley, 2010). Driving is a complicated task with subtasks running in parallel. During driving one might also encounter sudden changes (e.g. traffic jams, road blocks and detours). Driving thus relies heavily on driving experience, perceptual and cognitive abilities (Ross et al., 2014).

Certain characteristics, associated with ASD, might interfere negatively with driving. For instance, visual information processing problems can lead to atypical processing of road hazards (Sheppard, Ropar, Underwood, \& van Loon, 2010). Furthermore, individuals with ASD are limited in understanding and predicting others' behavior (Zalla, Sav, Stopin, Ahade, \& Leboyer, 2009), possibly causing inadequate judgments of other road users' behavior. A limited ability to plan and execute actions in response to environmental changes can cause a slowed driving style (Glazebrook, Elliott, \& Szatmari, 2008; Fournier, Hass, Naik, Lodha, \& Cauraugh, 2010). Executive dysfunction, reflected in limited self-monitoring, creativity, mental flexibility and planning abilities, can cause driving to be stressful and dangerous (Hill, 2004; Van Eylen et al., 2011). Mental inflexibility does not mean that youth with ASD are incapable of rule-learning, rather, switching between rules, or situations without specific instructions, can be problematic 
(Van Eylen et al., 2011; Brady et al., 2013). Their rule-bounded rigidity might be problematic when unexpected events occur (e.g., a road obstacle requires full-line-crossing). On the other hand, as safe driving depends on motivational factors (Hatakka, Keskinen, Gregersen, Glad, \& Hernetkoski, 2002), youth with ASD might follow traffic rules more strictly and adapt cautious driving styles, leading to decreased crash risks compared to non-autistic peers (Porter, 2011).

Although little research exists on the relation between ASD and driving, Cox and colleagues (2012) surveyed parents/caregivers of youth with ASD attempting, or previously attempting, to learn to drive. Questions addressed reasons for the driving status, driving experiences, the relation between ASD and driving, as well as teaching strategies (i.e., effective and ineffective) for learning youth with ASD to drive. Results showed that, in comparison to relatively easy driving skills (e.g., maintaining lane position), complex driving skills, such as merging into traffic or multi-tasking, were reported as most problematic for youth with ASD. Parents, and others involved in driving instruction (e.g., driver instructors), should be aware of certain ASD specific difficulties: interpreting the behavior of other road users, dealing with unexpected situations, and sustaining attention. This study, which is part of the ongoing "Yes I drive!" project, extends on Cox et al. (2012) by surveying driver instructors. Driver instructors are important sources of information and might be complementary to the opinion of parents/caregivers, by more objectively reflecting the teaching process.

\section{METHOD}

\section{Survey Development}

The introduction of the survey enclosed basic ASD information, aiding driver instructors to identify learner drivers with ASD; the remainder consisted of demographic questions, as well as open and closed questions addressing the relation between ASD and driving. Respondents were asked to respond to all questions with the answer that best suited their professional opinion. They were able to skip questions for which they did not have an answer. First, it was determined whether they encountered youth with ASD (e.g., 'How often do you provide driving lessons to youth with ASD?'). After which their advice on teaching youth with ASD to drive was queried (e.g., 'How can the current driving education be improved to better fit the needs of youth with ASD?'). Closed questions, describing possible perceptual, motor and cognitive problems related to ASD, were based on existing literature and the questionnaire by Cox et al. (2012).

Respondents were asked to indicate whether they thought specific characteristics, often associated with ASD, have an impact on driving ability (e.g., 'difficulties with motor planning'). The response scale consisted of five answering categories ranging from no impact to high impact. Examples were provided for each question.

\section{Recruitment and Respondents}

A web-based link was sent to driving schools in Flanders (i.e., the Dutch-speaking region of Belgium). From 144 driver instructors, 98 completed the questionnaire (dropout rate $=44 \%$ ). About $50 \%$ had experience with ASD, and were willing to complete the questionnaire. The final sample therefore consisted of 52 driver instructors (40 males), aged 31 to 65 (mean=50.10, SD= 9.10) with driver instructor experience ranging from 1 to 37 years (mean=15.79, $\mathrm{SD}=10.31)$. 


\section{RESULTS}

\section{Open Questions}

Conventional content analysis provided coding schemes for the open ended questions (i.e., coding themes were derived directly from the responses; Hsieh \& Shannon, 2005).

What stands out when youth with ASD learn how to drive? Personality: A recurring remark was a lack of initiative, interaction and/or empathy in youth with ASD. Some driver instructors however reported high motivation and perfectionism. Finally, some reported that youth with ASD can be overly busy whereas some reported extremely silent. Emotion: Some reported that youth with ASD are unconfident and extremely cautious, whereas others reported that that they are overconfident and incautious. A display of emotional reactivity, when youth with ASD received negative feedback, when sudden changes were encountered and when confronted with other drivers' traffic violations, was reported. Cognition: Typical cognitive characteristics of ASD were reported (i.e., problems with multi-tasking and self-regulation, slowed information processing, too focused on details). Difficulties with judging and reacting to traffic situations were also reported. Finally, it was reported that youth with ASD display abstract and rigid reasoning, and interpret conversations, instructions and logic literally. Learning: As reported, difficulties with complex situations and generalization of skills might be alleviated by providing structure, clarity, repetition, demonstration, and visualization. Some driver instructors also recommended shorter lessons, and/or a slower lesson pace, as youth with ASD usually need more time and might be quickly tired. Motor performance: Reports were made of rigid movements, motor tics and motor coordination difficulties.

Do you experience strong assets of youth with ASD while driving? Yes/no: Some driver instructors merely responded with 'yes' or 'no'. Personality: Some driver instructors considered perfectionism, conscientiousness, motivation and interest as strong assets of youth with ASD. A persistent and grateful personality was also reported. Emotion: Some driver instructors indicated that youth with ASD have a sensitive personality with an ability to relate to other road-users. One driver instructor reported the necessity of a personal bond with the learner driver in order to experience such strong assets of youth with ASD. Cognition: Youth with ASD were given credit for their concentration, memory, intelligence, perceptiveness, and the ability to notice details. Learning: Some driver instructors mentioned that youth with ASD are quick, systematic learners (i.e., structure is important) that require little explanation. They were also reported as being rulebound, displaying thorough knowledge of traffic rules and correctly executing learned materials.

What are needs of youth with ASD when learning how to drive? Instructor: It was advised not to dwell on the diagnosis but to treat them equal to other learner drivers. Concerning interaction with the learner driver, some driver instructors advised to nuance, display tranquility, patience and bi-directional trust, and avoid staring. An individualized approach and adapted communication (i.e., scarcity in words, neutral intonation, avoiding closed questions) were also considered important. Lessons: Again, reports were made of an increased number of lessons with a shorter duration, including additional rest periods, as well as structure, visualization, demonstration and repetition. Changes in teaching strategies were discouraged. The importance 
to focus on one task at a time, provide directed and concrete instructions, formulate specific goals, create familiarity (i.e., same instructor and vehicle) and reduce uncertainty (i.e., discuss possible scenarios), were reported. The use of motivational strategies, while avoiding negative criticism, was also advised. Environment: It was reported that, ideally, lessons should start at a practice court after which it is necessary to include plenty of practice in real traffic environments. A visit to the exam centre before the actual examination was considered useful. Others: An automatic gear was reported to reduce driving task complexity. Psychological tests were reported to be helpful for determining whether youth with ASD are capable of learning to drive.

How can the current driver education program be improved in order to better fit the needs of youth with ASD? I don't know: Some driver instructors merely responded with 'I don't know'. Instructor: The need for ASD-specific driver instructor courses was recurrently mentioned. As for teaching style, it was reported to base this on tranquility, patience, kindness, and coaching. Some driver instructors stressed cooperation, with for instance, parents, mentors or the educational system, as this should increase motivation and trust, and provide knowledge of diagnosis and severity. Lessons: Switches between instructors were discouraged while driving lessons tailored to the needs of the learner was encouraged. Again, an increased number of lessons with a shorter duration was recommended in which instructors provide structure and repetition, a combination of theory and practice, content beyond basic teaching packages, and an increase of practical exercises. Exams: Also suggested was the need for adapting driving exams, and cooperation with official test centers (e.g., hire professionals for teaching or exam admission). Others: It was recommended to start with driving simulation, and to drive with an automatic gear. Some driver instructors opted to provide learners with financial aids to pay for increased time and effort from the instructors.

\section{Closed Questions}

Respondents indicated whether specific characteristics, often associated with ASD, impact driving ability (Table 1). Items were all scored above average $(>3)$. The most problematic items were 'Difficulty with concentration/attention' (e.g., allocate attention to relevant sources), 'Difficulty with emotional self-regulation' (e.g., stress due to a busy traffic environment) and 'Difficulty with unexpected routine changes' (e.g., a detour on a normally familiar route). The least problematic were 'Difficulty with motor planning' (e.g., sequence of actions necessary to start to drive) and 'Difficulty with sensory overstimulation' (e.g., due to neon signs). 
Table 1. Responses to the closed questions $(0=$ no impact, $5=$ high impact $)$

\begin{tabular}{lcccccccc}
\hline Question & 1 & 2 & 3 & 4 & 5 & $\begin{array}{c}\text { Total } \\
\text { responses }\end{array}$ & Average & SD \\
\hline Difficulty with motor planning & 0 & 18 & 13 & 15 & 4 & 50 & 3.10 & 0.99 \\
Difficulty with multitasking & 0 & 7 & 20 & 13 & 10 & 50 & 3.52 & 0.95 \\
Difficulty with concentration/attention & 0 & 4 & 10 & 24 & 14 & 52 & 3.92 & 0.78 \\
Difficulty judging other's behavior & 0 & 4 & 14 & 20 & 12 & 50 & 3.80 & 0.82 \\
Difficulty with emotional self-regulation & 0 & 4 & 11 & 15 & 22 & 52 & 4.06 & 0.98 \\
Difficulty generalizing information & 0 & 8 & 15 & 18 & 10 & 51 & 3.59 & 0.98 \\
Difficulty with unexpected routine changes & 0 & 4 & 11 & 17 & 17 & 49 & 3.96 & 0.96 \\
Difficulty breaking traffic rules & 0 & 12 & 12 & 15 & 9 & 48 & 3.44 & 1.07 \\
Difficulty with other's braking traffic rules & 0 & 8 & 7 & 19 & 12 & 46 & 3.76 & 1.04 \\
& & & & & & & & \\
Difficulty with sensory overstimulation & 0 & 16 & 11 & 17 & 3 & 47 & 3.15 & 0.98 \\
\hline
\end{tabular}

\section{DISCUSSION}

This study surveyed driver instructors regarding the driver behavior of youth with ASD. Flemish driver instructors who encountered learner drivers with ASD acknowledged potential problems. When considering the open questions, the need for structure, clarity, visual demonstration, practice and repetition, and an individualized approach were recurrently noted. This coincides with literature describing benefits of structure, overview, clarity, imagery, concreteness, etc., for people with ASD (Cox et al. 2012; Van Eylen et al., 2011; Vermeulen, 2013). Responses were not always consistent, rather often contradicting each other. For instance, perfectionism was rated beneficial as well as detrimental for driving performance. The relation between ASD and driving performance might thus even be positive. This diversity between learner drivers supports the current classification of autism as a "spectrum" disorder with ASD specific and non-ASD specific characteristics (e.g., intelligence) varying from person to person, accounting for the variation in the capabilities and limitations of people with ASD (Grzadzinski, Huerta, \& Lord, 2013; Vermeulen, 2013). Strikingly, for the closed questions querying the impact of possible perceptual, motor and cognitive problems related to ASD, driver instructors never indicated a lack of impact on driving. This is different from the perspective of parents/caregivers in the study from Cox et al. (2012) where a lack of impact was provided in each of the ratings, of the impact of specific characteristics associated with ASD, on their son/daughter's driving skills. This study entailed some limitations. First, while this survey extended on Cox et al. (2012) by assessing the perspective of driving instructors, it will also be of interest to survey youth with ASD themselves and thereby get insights in their own perspectives of the matter. Second, there is a chance of misclassification and of under- or over-diagnosis by the driver instructors. More research on the relation between driving and ASD is therefore warranted. Third, although the closed questions queried the potential impact of difficulties often related to ASD, the questions did not query whether the driver instructors observed those difficulties in youth with ASD, nor did the questions specifically queried the relative impact of those characteristics for ASD. Therefore, driver instructors might have answered those questions generally, which might have caused the lack of impact for each item. 


\section{CONCLUSION AND IMPLICATIONS}

Similar to Cox et al. (2012), the results indicate that learning to drive presents a substantial challenge for youth with ASD. This survey provides relevant information for future research concerning the relation between driving and ASD. The results also entail some practical implications. For instance, financial aids and driver instructor courses might improve the accessibility of driving lessons for youth with ASD. Furthermore, although not specifically queried, driver instructors indicated driving simulation as a mean to familiarize ASD learner drivers with driving. Driving simulation has been proven to be a valid, safe and efficient method to assess and train drivers in a controlled manner, including a wide range of road and traffic conditions (Mayhew, et al., 2011; Reimer, et al., 2013; Rosenbloom \& Eldror, 2014). But research addressing driving simulation as a tool specifically for youth with ASD is still lacking. Another study (i.e., in the data collection phase) from the ongoing "Yes I drive!" project however will investigate hazard perception, and the underlying mechanisms (i.e., executive functioning and action observation), in youth with ASD, using a driving simulator, an eye tracker, and computer tasks (e.g., a computerized stop signal task). In the near future, the "Yes I drive!" project will also entail the development of driver training packages for youth with ASD.

\section{REFERENCES}

Brady, D. I., Schwean, V. L., Saklofske, D. H., McCrimmon, A. W., Montgomery, J. M., \& Thorne, K. J. (2013). Conceptual and Perceptual Set-shifting executive abilities in young adults with Asperger's syndrome. Research in Autism Spectrum Disorders, 7, 16311637.

Cox, N. B., Reeve, R. E., Cox, S. M., \& Cox, D. J. (2012). Brief Report: Driving and Young Adults with ASD: Parents' Experiences. Journal of Autism and Developmental Disorders, 42, 2257-2262.

Feeley, C. (2010). Evaluating the Transportation Needs and Accessibility Issues for Adults on the Autism Spectrum in New Jersey. 89th Annual Meeting of the Transportation Research Board, January, (pp. 10-14). Washington, DC.

Fournier, K. A., Hass, C. J., Naik, S. K., Lodha, N., \& Cauraugh, J. H. (2010). Motor Coordination in Autism Spectrum Disorders: A Synthesis and Meta-Analysis. Journal of Autism and Developmental Disorders, 40, 1227-1240.

Glazebrook, C. M., Elliott, D., \& Szatmari, P. (2008). How do Individuals with Autism Plan Their Movements? Journal of Autism and Developmental Disorders, 38, 114-126.

Grzadzinski, R., Huerta, M., \& Lord, C. (2013). DSM-5 and autism spectrum disorders (ASDs): an opportunity for identifying ASD subtypes. Molecular Autism, 4(12), 1-6.

Hatakka, M., Keskinen, E., Gregersen, N. P., Glad, A., \& Hernetkoski, K. (2002). From control of the vehicle to personal self-control; broadening the perspectives to driver education. Transportation Research Part F, 5, 201-215.

Hill, E. J. (2004). Evaluating the theory of executive dysfunction in autism. Developmental Review, 24, 189-233. 
Hsieh, H.-F., \& Shannon, S. E. (2005). Three Approaches to Qualitative Content Analysis. Qualitative Health Research , 15(9), 1277-1288. doi:10.1177/1049732305276687

Mayhew, D. R., Simpson, H. M., Wood, K. M., Lonero, L., Clinton, K. M., \& Johnson, A. G. (2011). On-road and simulated driving: Concurrent and discriminant validation. Journal of Safety Research, 42, 267-275.

Porter, E. (Red.). (2011). Handbook of Traffic Psychology. Elsevier Science.

Reimer, B., Fried, R., Mehler, B., Joshi, G., Bolfek, A., Godfrey, K. M., . . B Biederman, J. (2013). Brief Report: Examining Driving Behavior in Young Adults with High Functioning Autism Spectrum Disorders: A Pilot Study Using a Driving Simulation Paradigm. Journal of Autism and Developmental Disorders, 43, 2211-2217.

Rosenbloom, T., \& Eldror, E. (2014). Effectiveness evaluation of simulative workshops for newlylicensed drivers. Accident Analysis and Prevention, 63, 30-36.

Ross, V., Jongen, E. M., Brijs, T., Brijs, K., Ruiter, R. A., \& Wets, G. (2014). The relation between cognitive control and risky driving in young novice drivers. Applied Neuropsychology: Adult, 1-12. doi:10.1080/23279095.2013.838958.

Sheppard, E., Ropar, D., Underwood, G., \& van Loon, E. (2010). Brief Report: Driving Hazard Perception in Autism. Journal of Autism and Developmental Disorders, 40, 504-508.

Van Eylen, L., Boets, B., Steyaert, J., Evers, K., Wagemans, J., \& Noens, I. (2011). Cognitive flexibility in autism spectrum disorder: Explaining the inconsistencies? Research in Autism Spectrum Disorders, 5, 1390-1401.

Vermeulen, P. (2013). Brein bedriegt: Autisme en normale tot hoge begaafdheid (Vol. 1). Leuven: Acco.

Zalla, T., Sav, A. M., Stopin, A., Ahade, S., \& Leboyer, M. (2009). Faux Pas Detection and Intentional Action in Asperger Syndrome. A Replication on a French Sample. Journal of Autism and Developmental Disorders, 39, 373-382. 